\title{
The Role of Flow in Tree-hole Rescue Crisis Intervention and Emotional Catharsis
}

\author{
Wu JiaTong ${ }^{1, a}$ \\ ${ }^{1}$ Applied Psychology, Shanghai Normal University, Fengxian District, Shanghai, China \\ a15195218910@163.com
}

\begin{abstract}
Flow is the experience of working full time and enjoying the flow of your life, regardless of the rewards, like a flowing stream of water. Based on the literature review, this paper discusses the role of flow in the actual rescue work, combined with the actual work of the rescue mission in the tree hole baby crisis intervention and emotional counseling and accompanying. The effect of flow intervention is positive. It can reduce the rumination of young tree-hole babies, increase and enhance their sense of life meaning and subjective well-being. Since the term "Flow"is rarely used in the process of intervention, this paper also looks forward to the further application of flow intervention.
\end{abstract}

Keywords: flow experience, crisis intervention, ruminant thinking, sense of meaning of life; tree-hole action

\section{心流在树洞救援危机干预及情绪疏导中的作用}

\author{
吴珈䑣 1 , a
}

\author{
${ }^{1}$ 上海师范大学应用心理学系, 奉贤, 上海, 中国 \\ a15195218910@163.com
}

\section{摘要}

心流是指当人们废寝忘食，不计回报地全身心投入工作，并且乐在其中时的体验，这种体验就好像流动的水流 一样，源源不断、顺畅不息。本文通过文献回顾，结合在树洞行动救援团对树洞宝宝开展的危机干预和情绪疏 导、陪伴等实际工作，探讨心流在实际救援工作疏导陪伴干预环节中的作用。心流干预的效果是积极的，可以 减少青年树洞宝宝的反刍思维，增加和提升他们的生命意义感和主观幸福感。由于在干预过程中很少用到心流 这一说法，本文也展望了心流干预的进一步应用。

关键词: 心流体验，危机干预，反刍思维，生命意义感，树洞行动

\section{1. 心流的概念及应用}

我们一定有过这样的经历: 因为做事太过于投入, 忘记了时间的流逝, 甚至感觉不到自己的存在, 进入 一种 “浑然忘我” 的状态, 甚至为它废寝忘食, 不计 回报并且乐在其中。在这样的状态中，我们会获得内 心的秩序与安宁, 产生极大的满足感, 从而感到快乐。 这样的活动因人而异, 可以是攀岩、国际象棋、体育 运动、艺术活动 (如美术、舞蹈、歌唱) 、文学创作 和各种极限冒险运动等。并且, 我们在活动中只关注 活动本身, 沉浸并享受在活动过程中, 不求外部回报 或结果。

这种现象在心理学中则叫做心流体验（flow experience），简称 “心流” [1]。心流是对那些可以 意会不能言传的全神贯注、乐此不疲、沉浸、专注、 神入等心理过程的一个概念化。在心流体验中, 个体 的主观状态是愉悦的、主动的、忘我的，又体现出活 动的即时性、连续性和内在的目的性 ${ }^{[2]}$ 。

心流是由条件状态到体验状态的过程, 条件状态 不成立，心流也就无法产生。首先，心流的条件状态 的特征之一是, 这个任务必须要在我们熟悉的技能和 挑战之间进行匹配平衡, 当任务挑战性与个体本身技 能水平相平衡时, 个体就有机会进入心流状态, 也就 是说这个任务既要是我们能掌握和控制的, 又要是具 有挑战性的。如果挑战太难, 我们就会进入一种情绪, 叫焦虑, 如果挑战太容易, 我们就会进入另一种情绪, 
叫无聊。处于焦虑和无聊之间, 就容易进入心流状态。 心流的这一特征能让我们获得成就感; 其次, 心流的 条件特征之二是目标的明确、清晰性, 这一特征能让 我们获得控制感; 最后，心流体验需要及时反馈，当 手头任务得到明确并且及时的反馈和判断的时候, 会 有助于人们针对不断变化的情况, 快速调整自己的表 现来维持心流状态 ${ }^{[3]}$, 并且这些反馈通常是正面奖赏, 能够激发我们心中的奖赏机制, 增加我们的愉悦情绪。

既往研究表明, 心流体验在促进个体心理健康和 主观幸福感方面发挥着重要作用。心流能给人带来积 极的情感体验, 提高学习或工作的创造力水平, 规律 性地进入心流状态是人们幸福感的关键组成部分 ${ }^{[3]}$ 。 Asakawa 对日本大学生的心理健康指数进行调查, 结 果发现处于心流体验中的大学生其心理健康指数更 高 ${ }^{[4]}$, 因为心流体验中的个体会报告更多的积极情绪; Hirao 等人发现心流体验的频率与心理健康之间正相 关关系, 提出心流体验可以提高心理健康水平 ${ }^{[5]}$; 此 外, 在一项 “慢性疼痛人群的心流体验” 研究中, 通 过研究证实心流是一种积极的心理状态, 处于心流中 的人更容易体验到愉悦感、高自尊、成就感, 强内在 动机和生活满意度, 更容易专心投入积极的 “情感获 益”中, 从而从慢性疼痛的痛苦中转移出来 ${ }^{[6]}$ 。

目前研究心流所用到的研究方法主要是访谈法、 问卷调查法、心流体验抽样法和实验法。访谈是了解 心流体验最常用的方法, 也是较传统的方法, Csikszentmihalyi ${ }^{[1]}$ 最初就是通过访谈许多有类似 体验的人群而提出的心流概念。这一方法最大特点是 可以在真实生活情境中细致地了解被试, 确定心流体 验的动力学特征和大致范围, 但其缺点是, 个体主观 感觉往往不准确, 容易出现实验者效应, 且访谈时间 较长, 效率较低; 问卷调查是测量心流体验较常用的 方法。Nakamura 认为问卷调查主要用于测量被试心流 体验的维度、情境及个体间差异 ${ }^{[7]}$, 现沿用的主要是 Jackson 和 Mar sh ${ }^{[8]}$ 编制的心流体验状态量表 (FSS); 心流体验抽样法的核心在于多次重复评估个体在日 常生活中对不同行为与环境的感受, 以保证结果的客 观性和准确性; 实验法也就是心理学研究最常用的一 种方法, 研究者设计一系列实验和准实验来研究被试 在参与实验前后的心流体验。

我们过往心理学的研究大多倾向关注如何摆脱 人类劣势, 而积极心理学作为时代发展下的新式学科, 倡导心理学的积极取向, 以研究人类的积极心理品质、 关注人类的健康幸福与和谐发展, 从而使不好的感受 和行为减少。心流是积极心理学中一个重要概念, 具 有积极心理学的特性, 必须为全人类的生活服务, 必 须扎根于人类的生活实践，必须为增进全人类的幸福 而提供技术支持 ${ }^{[9]}$ 。因此在危机干预中值得关注。然 而目前心流的应用研究主要集中在教育、网络成瘾和 企业管理等领域, 很少有在危机干预中应用的研究。 因此我们以下从理论角度和个案干预实践角度, 具体 探讨心流在线上危机干预疏导陪伴环节中可以发挥 作用的原因。

\section{2. 树洞救援工作及减少思维反刍的必要性}

危机干预, 是一种通过调动处于危机之中的个体 自身潜能, 来重新建立或恢复危机爆发前的心理平衡 状态的行为 ${ }^{[10]}$ 。“树洞行动” 中的树洞是传说古时候, 心里藏着秘密又希望倾诉的人, 跑到森林里找一个树 洞对其倾诉秘密。在当代, 人们常在网络社交媒体上 倾诉自己的想法, 社交媒体 (如微博) 可以说是现代 化的 “树洞”。2018 年 7 月 27 日, 以荷兰阿姆斯特 丹自由大学黄智生教授发起的 “树洞行动” 救援团, 通过人工智能高科技手段分析网络社交媒体信息, 已 经对大量系统发现的高风险自杀人群进行网络自杀 救助, 暂时阻止了他们的自杀行为, 并进行后续的疏 导陪伴工作, 带来了明显的社会效益。此外, 作为树 洞行动救援团的成员，我们亲切地称这些检索出来的 人群为“树洞宝宝”。

自杀是一个全球性的心理健康问题 ${ }^{[11]}$, 在树洞救 援的过程中, 我们不禁思考, 导致如此多的树洞宝宝 采取自杀的原因是什么? 在我们的主动干预和交流 陪伴过程中, 发现他们大多数经历着抑郁症或抑郁状 态的困扰，而导致他们抑郁或他们抑郁之后会采取的 一个关键的心理过程或行为, 就是反刍, 反刍思维是 个体将注意集中在痛苦的情况或事件的原因和影响 上的一种消极重复性思维 ${ }^{[12]}$, Nolen-Hoeksema 表明了 反刍思维和抑郁症有密切的关系 ${ }^{[13]}$, 具体来说就是反 刍思维会加重抑郁症持续时间和症状。并且, 当前的 研究已经证实, 反刍思维者存在对消极刺激的注意偏 向, 并且这种注意偏向会加重个体的反刍思维, 最后 导致抑有的发生，同时，大量点探测范式实验结果表 明抑郁焦虑个体比正常个体更容易将注意投放到消 极情绪信息中去 ${ }^{[14]}$, 因此抑郁与反刍呈双向因果关系, 更容易导致恶性循环, 使个体陷于抑郁情绪中不能自 拔。

研究也发现, 反刍思维和自杀风险密切相关, 例 如 Rory 等人的研究发现反刍思维能够增加自杀意念 ${ }^{[15]}$; Cole 等人发现反刍思维通过增加个体的感知压力 水平, 进而影响自杀意念 ${ }^{[16]}$ 。因此, 反刍思维不仅会 加重抑郁时间和症状, 还能提高自杀风险, 而抑郁症 患者本身就有一定的自杀风险, 抑郁可能是反刍思维 与自杀的中介, 因此对反刍思维在其与抑郁和自杀方 面的研究有利于我们寻找纾解抑郁情绪、挽救生命的 有效干预措施。

而在我之前的若干次树洞救援经历中, 由于接触 到的需要紧急自杀危机干预的树洞宝宝并不多, 大部 分时候都是对他们进行陪伴干预, 即线上疏导他们的 情绪和状态, 以劝说、倾听和建议为主。总结发现, 几乎所有患有抑郁症的树洞宝宝都不可避免地频繁 出现反刍思维, 这导致他们深陷负性事件和抑郁情绪 之中无法自拔, 甚至出现自杀意念。而从个案干预实 践中来看, 心流在线上疏导所起的效果是积极的。主 要原因可能是心流的一个突出特点是完全沉浸, 即当 我们处于心流体验中的时候, 注意力高度集中, 对自 
己正在做的事情充满热情，具有自激励性 ${ }^{[3][17]}$ ，在这 之中心流体验积极的意义不是一个固定结果和最后 结局，而是一个行为过程，包括过程的体验，个体的 认知、情绪和行为, 因此, 当树洞宝宝用心去做某件 事的时候, 反刍思维就会减少, 消极的情绪就会减弱, 积极的态度相应增长, 因而化解了自杀危机或抑郁情 绪。

\section{3. 心流作用}

生命意义感指个体感知自身存在的重要性, 理解 并追寻生命存在的目的与意义, 以实现自我的生命价 值 ${ }^{[18]}$ 。过去有研究表明, 生命意义感与自杀意念存在 显著负相关, 并且生命意义感能显著负向预测自杀意 念 ${ }^{[19]}$ 。生命意义感对心理健康还具有调节作用, 在应 激条件下能够调节抑有情绪和一般健康问题 ${ }^{[20]}$ 。缺乏 生命意义感的个体倾向于将死亡视为逃离痛苦人生 的方法, 甚至认为是唯一的解脱方法 ${ }^{[21]}$, 说明缺乏生 命意义感可能产生心理问题, 甚至导致自杀, 因此可 以发现, 青年的生命意义感越高, 自杀和抑郁水平和 自杀意念水平也可能越低。

主观幸福感是个体依据其主观标准对自己生活 质量的整体感受和判断, 具有主观性、稳定性和整体 性等特性 ${ }^{[22]}$, 由对生活的满意、积极情感的体验和消 极情感的缺乏所构成, 个体对整体生活的满意程度越 高, 体验到的积极情感愈多消极情感愈少, 则个体的 幸福感体验愈强烈, 它是衡量个体生活质量的重要的 综合性心理指标。有研究表明, 生命意义感能预测主 观幸福感, 开展生命意义感教育是提升主观幸福感的 重要途径 ${ }^{[23]}$ 。而大量研究表明主观幸福感越高, 抑郁 水平越低 ${ }^{[24][25][226]}$, 因此青年生命意义感、主观幸福感 和抑郁之间有着强烈的相关联系, 如何提升青年的生 命意义感和主观幸福感就成为关键问题。

综上所述我们可以发现, 生命意义感能够提升个 体的心理健康水平和主观幸福感, 并且生命意义感和 主观幸福感又能降低个体的消极情绪, 同时前文又提 到抑郁发生的机制可能是个体的反刍思维, 而心流的 本质则是从积极心理学角度给人带来积极的情感体 验, 降低抑有和自杀意念水平。由此我们可以推测, 疏导陪伴干预中的心流策略能够减少青年树洞宝宝 的反刍思维, 增加和提升他们的生命意义感和主观幸 福感, 从而减少他们的抑郁情绪和自杀意念。

\section{4. 疏导策略}

前文已经提到, 心流指的是当人们沉浸在手头的 某件事情或某个目标中时, 所体验到的一种全神贯注 投入并乐在其中的精神状态。但是, 心流状态难得一 遇, 尤其是在危机干预中, 寻找让树洞宝宝进入心流 状态以达到缓解抑郁情绪和自杀意念的策略并非易 事。根据以往我与树洞宝宝的疏导陪伴经验以及对于 一篇由心理健康老师在危机干预中使用到的心流策 略的文章的参考 ${ }^{[10]}$, 并且针对树洞救援的网络性质、
主动疏导、长期陪伴等实际, 我总结下来心流策略在 线上疏导干预中的应用涉及以下步骤:

(1) 获得联系后, 尊重隐私, 尝试通过其他方式 来识别树洞宝宝的自杀风险, 帮助他们理解自己自杀 的动机。

(2) 暂时缓解自杀危机后, 认真倾听他们并表示 理解, 逐渐获得他们的信任, 从而让他们渐渐打开心 扉。

(3) 获得信任和建立一定联系后, 可以通过两种 方式帮助树洞宝宝获得心流体验, 第一种方式是, 通 过聊天或通话向他们抛出一些有逻辑性、有猜测性、 神秘性的问题 (一般是要根据树洞宝宝的自身情况如 兴趣爱好、学习情况、擅长领域等进行探讨) 以此来 简洁、直接地帮助他们创造一种专注进行某行为时的 心理状态, 在此状态时, 他们通常情绪点会慢慢调高, 不愿被打扰，不愿意被中断，注意力会从最初的 “活 着没有意义” 的沉浸式痛苦, 转移到一些现实或理想 性的话题上面去。

（4）由于第一种方式获得的心流体验是建立在 双方交流上的，因此比较短暂、不易持久，但是见效 快、效果强，因此在一段时间的第一种方式的实行之 后，陪伴的这位树洞宝宝的情绪和状态都有所改善的 时候，则在第一种方式的基础上采用第二种方式，即 帮助树洞宝宝找到并培养长期的心流体验，鼓励他们 发展曾经的兴趣爱好或培养新的兴趣爱好, 找到自己 真正喜爱的事情, 并且可以和他们一起制定计划, 一 起投身这一爱好, 这些活动可能是象棋、登山、摄影、 绘画、唱歌、写作、阅读等。要做到这一点也需要提 升树洞宝宝自身的心流力, 即不再局限在心流条件的 限制上, 而是主动去改变或扩大条件, 例如在没有得 到及时反馈的情况下依然能保持心流等。第二点实施 起来绝非易事，虽然实行成功会让树洞宝宝获得极大 的生命意义感和幸福感，但是如果施行不顺，方法不 对, 就有可能导致树洞宝宝会觉得自己没有能做的事 情, 反而起到相反的结果, 所以第二种方法要谨慎使 用, 一般需要保证树洞宝宝的情绪状态比较稳定, 并 且自己也有比较大的意愿想要改变的时候才会使用。

(5) 最终的目的是让树洞宝宝发现自己认知的 片面性, 即自己并非是一无是处或是生命无价值无意 义的, 当他们在心流状态中去思考和观察时, 会全情 投入，消极情绪减弱，危机事件也会由此转危为机。

下面我们来看两个具体案例。

案例 1: 树洞宝宝 A（下文简称 A）与我一开始交 流时经常提到 “没办法” “我真没用” “生命没有意 义” 等无奈绝望的话语, 在我耐心倾听、安抚了她的 情绪后, 我问她是否可以打电话。她犹豫了一下答应 了。我耐心地和她说话, 起初她说话好像比较艰难, 话很少, 都是我问她答, 渐渐地, 她因为足够信任了 我也可能是因为她的话匣子被打开, 接着和我回忆起 她在公司遇到的趣人趣事，讲到很多细节，对于不懂 
的细节我也会问她, 并且给她反馈“是……这样对吗”, 这样让她更有兴致说下去了。时间一分一秒过去, 不 知不觉就聊了两个多小时, 她说她现在有心情出去吃 个饭, 我感到很欣慰。我想, $\mathrm{A}$ 在交流的过程中, 讲 述自己事情的时候进入了一种专心回忆的心流状态, 是完全沉浸不愿被打扰的, 这一体验让她的大脑活跃, 暂时缓解了抑有情绪, 并且有动力去进行一些社会活 动。

之后, 她的情况已经好很多了。她经常把她写的 小说给我看, 为了鼓励她, 我和几个老师找到一些公 众号和写文的工作推荐给她, 希望她能以此继续做自 己喜欢的事并且也能得到一份收入, 增加自己的价值 感。

在这个案例中, 回忆过去的事情是在聊天中建立 的短暂的心流体验, 能快速直接地缓解抑郁情绪和自 杀意念; 写作是树洞宝宝 A 长期稳定的心流体验来源, 能够帮助她逐渐建立生命意义感，提升主观幸福感。

案例 2: 树洞宝宝 B (下文简称 B) 最初是因为情 伤而经常自伤, 与我交流时经常提到 “没人会喜欢有 抑郁症的人” “我好后悔”之类的话, 在通过倾听劝 慰暂时平复她的情绪和建立初步信任和联系之后, 我 发现她是个很聪明很善于思考的女孩子, 这次我没有 让她回忆过去的痛苦回忆, 而是和她讨论一本书叫做 《庞思琪的初恋乐园》, 讨论这本书中女孩的事情, 讨论作者林奕含的故事和选择, 讨论有关女性、性侵、 教育、人性。在我们的讨论、互相表达观点的过程中, 我发现她的逻辑越来越清晰, 思考越来越全面, 思绪 也慢慢开阔明朗起来, 她后来和我说这样的讨论让她 感受到思维的乐趣, 感觉到自己是活着的。B 在这样 的讨论中获得了即时的愉悦性高峰体验。

之后, 我发现她的性格里有一种激烈、热情的东 西, 并且发现她喜欢写诗和画画, 便说想看她的作品, 她也很乐意地给我看, 我认真地看了并给出了积极、 具体的评价, 这样更加增加了她分享的欲望。之后我 们也经常聊天, 了解到她是个大学生, 经常拿奖学金, 并且也渐渐获得了良好的人际关系, 在和同学、朋友 的交往中获得了支持与爱。在这过程中, $\mathrm{B}$ 的心流体 验十分明显, 她更倾向于是在交流和分享中产生心流, 这极大地增加了她做事的动力和继续活下去的希望。

\section{5. 展望}

心流研究有助于积极心理学理论的完善和构建, 增加个体更多的愉悦体验, 让人们对从事的活动充满 兴趣。日后对心流体验的研究, 有必要将访谈法、问 卷调查法与心流体验抽样法相结合, 以获得更全面、 准确、生动的原始资料。

心流体验日后的研究将朝着以下方向努力: 第一, 心流体验研究将逐渐走进实验室, 尤其是利用各种实 验设备进行研究, 如 ERP、PET、fMRI 等; 第二, 针对 该理论过于西方化的特质, 应加强对心流体验的跨文
化研究; 第三, 应将心流体验理论与人们的日常生活 更紧密联系起来, 为增进全人类的幸福提供技术支持 ${ }^{[2]}$ 。最后一点与自杀危机干预息息相关, 目前尚没有 理论或实证研究探讨心流在危机干预中的策略应用, 结合树洞救援团的网络和长期干预性质，未来可以展 开对线上危机干预中的心流策略的探究, 如可以依据 心流以往的研究, 设计本文的心流策略在自杀危机干 预中的具体方案, 论证后, 开展对照实验, 验证效果; 也可以在已经开展的案例中, 用访谈的方式来收集案 例, 进行叙事研究。

\section{6. 结论}

(1) 通过文献回顾推测, 心流体验可以减少个体 的反刍思维, 增加和提升个体的生命意义感和主观幸 福感。

（2）从个案干预实践来看, 心流策略在树洞救援 危机干预中的效果是积极的, 主要以情绪疏导的形式 呈现。

\section{项目基金}

本文为树洞行动救援团第三期培训的结 业论文。

\section{REFERENCES}

[1] Csikszentmihalyi, M., LeFevre, J. (1989) Optimal experience in work and leisure. Journal of personality and social psychology, 56: 815-822.

[2] Li, C. (2017) A review of the research on flow experience. Journal of the Kaifeng Institute of education, 37: 187-189.

[3] Han, S.Y. (2018) How do you get a flow experience? Shanghai education, 6: 11-12.

[4] Asakawa, K. (2004) Flow experience and autotelic personality in Japanese college students: how do they experience challenges in daily life? Happiness Stud, 5: 123-154.

[5] Hirao, K., Kobayashi, R., Okishima, K., Tomokuni, Y. (2011) Influence of flow experience during daily life on health — related quality of life and salivary am ylase activity in Japanese college students. Jpn. J. Occup. Med. Traumatol, 59: 13-18.

[6] Katie, R., Norelee K., Dominic, H. (2012) The flow experiences of people with chronic pain, occupation. Participation and Health, 32: 104-112.

[7] Nakamura, J., Csikszentmihalyi, M. (2002) The concept of Flow. In: The handbook of positive psychology. New York. pp. 89-103.

[8] Jackson, S.A., Marsh, H.W. (1996) Development 
and validation of a scale to measure optimal

experience: the Flow State Scale. Journal of Sport and Exercise Psychology, 18: 17-35.

[9] Ren J., Shi J., Ma T.Y. (2009) Overview of the Flow study. Advances in psychological science, 17: 210217.

[10] Zhang X.D. (2021) Creating flow experience and restoring life confidence-the application of positive psychology in student psychological crisis intervention. Friends of Head Teachers (secondary school edition), 7: 36-37.

[11] Sha F., Yip P.S., Law Y.W. (2017) Decomposing change in China's suicide rate, 1990-2010: Ageing and urbanisation. Injury Prevention Journal of the International Society for Child \& Adolescent Injury Prevention, 23: 40-45.

[12] Grafton, B., Southworth, F., Watkins, E., MacLeod, C. (2016) Stuck in a sad place: Biased attentional disengagement in rumination. Emotion, 16: 63-72.

[13] Nolen-Hoeksema, S. (1991) Responses to depression and their effects on the duration of depressive episodes. Journal of Abnormal Psychology, 100: 569-582.

[14] Mathews, A., MacLeod, C. (1985). Selective processing of threat cues in anxiety states. Behavior Research and Therapy, 23: 563-569.

[15] Rory, C., Connor., Noyce, R. (2008) Personality and cognitive processes: Self-criticism and different types of rumination as predictors of suicidal ideation. Behaviour Research and Therapy, 46: 392-401.

[16] Cole, A.B., Wingate, L.R.R., Tucker, R.P. (2015) The differential impact of brooding and reflection on the relationship between perceived stress and suicide ideation. Personality and Individual Differences, 83: 170-173.

[17] Chen, X. (2014) Flow experience and its research status. Journal of the Xuzhou Normal University (philosophy and social sciences), 40: 150-155.
[18] Liang, J.L., Cui, X.L., Wu, H.T., Peng, L.X. (2018) Development and reliability and validity of sense of life scale for college students. China Health Administration, 358: 303-307.

[19] Xu, L.X., Shi, Y.S., Xiong, C,E., Liu, Z.S., Huang, C.P., Zeng, D.Z., Meng, S.H. (2019) Effect of sense of meaning of life on suicidal ideation in patients with depression. Hainan medicine, 21: 2733-2736.

[20] Li, H. (2006) The moderating effect of selftranscending meaning of life on the relationship between stress and health. Journal of Psychology, 38 : 422-427.

[21] Xu, J., Chang, M.L. (2011) The relationship between life meaning and death attitude of college students. Journal of Zhangzhou Teachers College (Natural Science Edition), 29: 128-132.

[22] Deiner, E., Suh, E., Lucas, R.E., Smith, H.L. (1999) Subjective well-being: Three decades of progress. Psychological Bulletin, 125: 276-302.

[23] Hou, X.L., Hu, T.Q., Xie, Q. (2016) The relationship between life meaning and subjective well-being in adolescents: the mediating role of self-efficacy. Psychological techniques and applications, 4: 738743.

[24]Wang, J., Liu, S.M., Zhang, P.C., Wu, H.Y., Chi, X.L. (2016) Effect of positive psychological intervention on emotion and subjective well-being of college students with depressive symptoms. Special Education in China, 11: 44-50.

[25] Wu, X.L., Gai, X.S. (2018) Two-way relationship between adolescent well-being, depression and academic achievement: A cross-lagged study. In: 21th national academic conference on Psychology. Beijing. pp. 395-396.

[26] Zhang, Q., Liu, J.X., Chen, C.W., Li, Y.X. (2020) The effect of depression on subjective well-being: the mediating effect of resilience. Vocational education, 07: 111-114. 